\title{
Purpuric Rash of Meningococcemia
}

\author{
Vanessa London \\ Paul Aronowitz \\ Internal Medicine, California Pacific Medical Center, San Francisco, California
}

\begin{abstract}
A previously healthy 62-year-old man presented to the emergency department with 4 days of headache, fever, and chills. Within several hours of presentation, he developed septic shock. His temperature was $39^{\circ} \mathrm{C}$, and his white blood count was $17,000 / \mathrm{mm}^{3}$ with $38 \%$ bands. He had acute renal failure (creatinine $=2.5$ ) and mild mental status changes. His blood pressure decreased from $103 / 63$ to $71 / 46$ and was not responsive to intravenous fluid administration. He therefore was begun on pressors. Eight hours after arrival, a petechial and purpuric rash suddenly appeared on the patient's extremities, including the palms of his hands (Fig. 1). Ceftriaxone was added to his initial antibiotics (vancomycin and pipercillintazobactam), and he was treated with stress-dose hydrocortisone and activated drotrecogin alpha. The following day, blood cultures grew gram-nega-
\end{abstract}

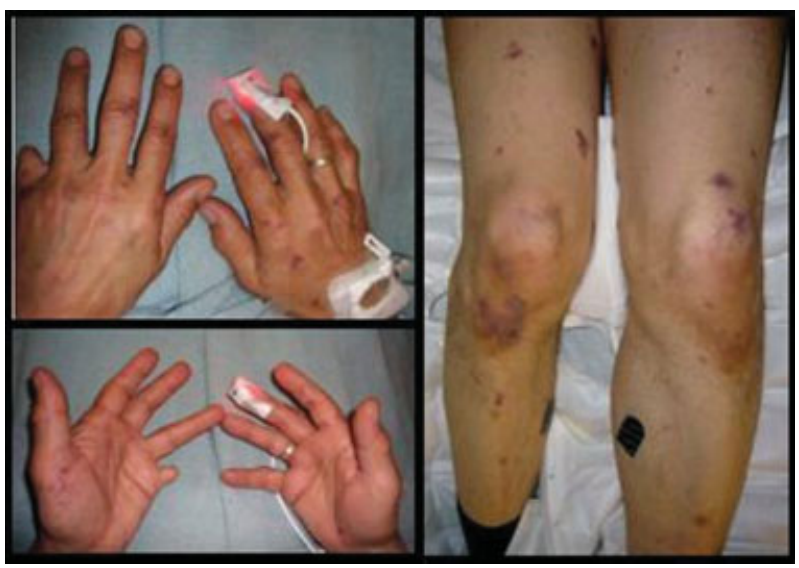

FIGURE 1. Day of admission. tive diplococci in pairs, and preventive measures were taken, including treating close contacts and placing the patient under droplet precautions. The cultures eventually confirmed Neisseria meningitidis. The patient made excellent progress-his mental status improved, he was weaned off pressors after 3 days, and his renal failure resolved. Figure 2 demonstrates the evolution of the purpuric lesions, which became more prominent as he otherwise made clinical improvement. He was discharged home after 2 weeks of intravenous ceftriaxone in good condition.

Address for correspondence and reprint requests: Vanessa London, 19523 Ballinger St., Northridge, CA 91324.

Received 23 July 2007; revision received 22 August 2007; accepted 25 August 2007.

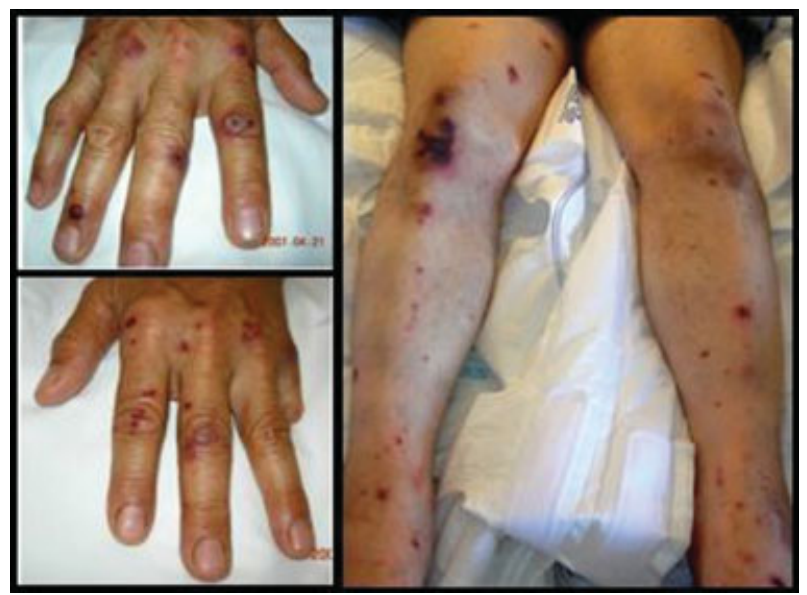

FIGURE 2. Hospital day 7 . 